\title{
An Evaluation of MPLS-TE Based PMIPv6 Network
}

\author{
Hatsadin Payappanon, Thossaporn Kamolphiwong, and Robert Elz
}

\begin{abstract}
This paper gives some results for a simulation of a Proxy Mobile IPv6 (PMIPv6) network based upon Multi-Protocol Label Switching with Traffic Engineering (MPLS-TE) for handling packet forwarding through the network and handling link or node failure. These results are compared with results from a simulation of a standard PMIPv6 network. The simulation uses the OMNET++ network simulation framework. This paper represents work that remains in progress, with more scenarios yet to be tested. However, the tentative results so far indicate that the MPLS-TE/PMIPv6 combination outperforms standard PMIPv6.
\end{abstract}

Index Terms-MPLS-TE, LSP, PMIPv6 and IP-in-IP.

\section{INTRODUCTION}

The Internet protocols were designed to use a single identifier, the IP address, to both identify a node to its peers, and to allow the network to locate the node and deliver data to it. This poses a problem for devices that move around the network, yet want to appear to other nodes as a single unchanging device, rather than a series of different devices with different addresses. The rapid growth of use of mobile devices makes providing good solutions to this problem more urgent that it was earlier.

For the next generation Internet, the protocols developed to deal with this issue include Mobile IPv6 (MIPv6) [1], Fast-MIPv6 (FMIPv6) [2], and Hierarchical-MIPv6 (HMIPv6) [3] and others more. The older Internet protocols (IP version 4) [4] have similar mechanisms [5]-[7]. These protocols provide a host based mobility management scheme that requires all mobile devices to participate in the signaling management required to allow the node to move while retaining its identity. For optimum performance, all nodes on the network must participate. The effect upon the mobile nodes is to increase their complexity, and also perhaps reduce battery life due to the requirements of the mobility protocols.

An alternative scheme has been created to allow for the common case of a mobile node that moves mostly within the limits of its parent network. This is Proxy Mobile IPv6 (PMIPv6) [8] which is a network-based mobility management protocol. That is, the network takes care of managing mobile nodes, rather than the nodes themselves, simplifying the software demands upon the mobile nodes. In a PMIPv6 domain, each mobile device communicates with its peers across the PMIPv6 domain via a tunnel between a Mobile Access Gateway (MAG) which is directly connected to the mobile node, and a Local Mobility Anchor (LMA) which

Manuscript received September 10, 2013; revised December 18, 2013. The authors are with Centre for Network Research, Computer Engineering Department, Faculty of Engineering, Prince of Songkla University Songkla, Thailand (e-mail: boy.hatsadin@gmail.com). provides the domain's visibility to the rest of the network.

Evaluation of the performance of mobility protocols concentrate upon two main aspects of the protocols. The overheads added to regular data flow to a node that has moved, and the delays involved in re-establishing connectivity after a node moves. There has been some recent works to provide mechanisms to reduce the handover delay [9], [10], and in particular, Astudillo et al. [11] proposed use of a label switched path (LSP) tunnel, as used in multi-protocol label switching (MPLS) networks [12] instead of the traditional IP-in-IP tunnel of PMIPv6. This is intended to reduce handover delay, and hence lost data packets during the handover period, and also reduces handover overheads, as well as reducing data transfer overheads.

Astudillo's work assumes the mobile node will connect to a MAG at its new location, perform the necessary network acquisition tasks, including securely proving its identity, the MAG would then send a binding update to the LMA, which would respond by creating a LSP tunnel to the MAG, which, given these tunnels are unidirectional, would also be creating a tunnel to the LMA.

We proposed [13], that gives the existing relationship between MAG and LMA, in that they are part of the infrastructure of the PMIPv6 domain, that the LSP tunnels between them could be pre-established, so that they are available for immediate use once the LMA has received the binding update from the MAG And we also proved that using the pre-configured LSP tunnel reduced the time spent in the handover period.

This research is continuation of [13] and investigates more scenarios in order to evaluate the impact of introducing the LSP tunnels into the PMIPv6 network in more realistic world. The proposed network is simulated using the OMNET++ Network Simulator [14] which provides detailed performance analysis of the quantitative performance parameters like end-to-end delay which the source and destination are in the same network and also analysis the time to recover the network when link failure occurs within the network.

The remainder of this paper is structured as follows. The next section provides some background information on PMIPv6 and MPLS-TE. Section 3 presents our network designs and the scenarios to be simulated, along with explanations. Section 4 presents the results of the simulations performed to date, with an analysis of these results. Section 5 provides some conclusions, and indicates what work remains to be done.

\section{BACKGROUND INFORMATION}

To understand our simulation and evaluation of the MPLS-TE based PMIPv6network, some basic knowledge of Multi-protocol Label Switching technology and Proxy 
Mobile IPv6 technology is required. The following two sections provide that background.

\section{A. Proxy Mobile IPv6}

Proxy Mobile IPv6 (PMIPv6) [8] is a variant of the Mobile IPv6 protocols [1] that allows for a node to move within a localized domain without requiring any signaling between the mobile node and its home agent. The most significant components in a PMIPv6 domain are the Local Mobility Anchor (LMA) and the Mobility Access Gateway (MAG). The LMA is responsible for maintaining the mobile node's reachability via communications to the appropriate MAG. The MAG is responsible for detecting movement by a mobile node, and initiating a binding registration with the LMA.

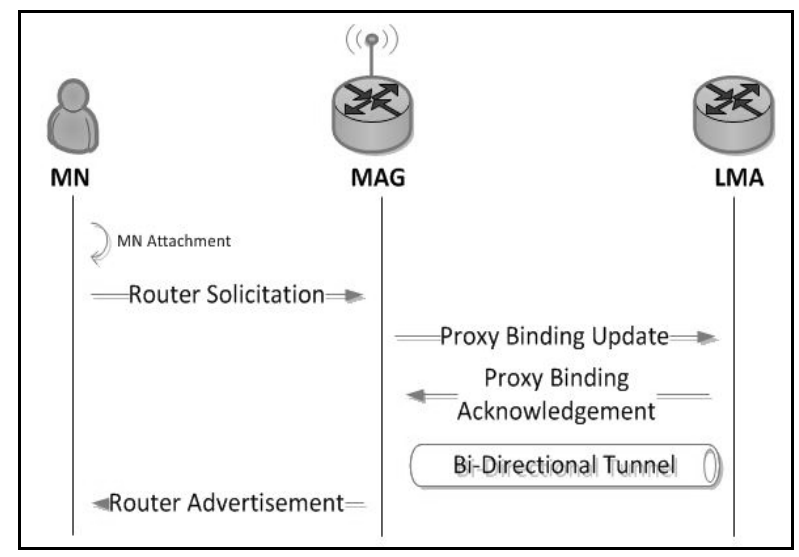

Fig. 1. Handover procedures in proxy mobile IPv6 domain.

When any IPv6 node detects the presence of a new link, it attempts to obtain networking parameters for that link by sending a Router Solicitation (RS) to any routers that are connected [15]. If the router that receives the RS is a MAG, it will initiate the mobile node hand over procedure by sending a binding update to the LMA as illustrated in Fig. 1. The LMA then creates a binding cache entry and responds with a proxy binding acknowledgment message which includes the appropriate home network prefix for the mobile node attaching. The MAG then creates a binding update list and sends a Router Advertisement (RA) message to the mobile node $(\mathrm{MN})$ containing the MN's home network prefix. The MN sees the same RA advertised network prefix from any MAG to which it connects, thus believes that it is simply reconnecting to the same network. The MN configures its address based upon its home prefix in the normal way [16]. Communications between the $\mathrm{MN}$ and any peer nodes then operate via the MN to MAG link, over a tunnel between MAG and LMA, and then using regular IPv6 forwarding to or from the peer node.

\section{B. Multi-Protocol Label Switching with Traffic Engineering}

Multi-Protocol Label switching (MPLS) [17] is a highly scalable technique that is widely used in the core network [18]. It implements packet forwarding using a label attached to each packet to assist with the forwarding decisions. MPLS introduces the Label Switched Path (LSP) tunnel which provides the mechanism to transport labeled data packets from the source node along the path to the destination node. There are three components in an MPLS network, ingress and egress Label Edge Routers (LER), and Label Switch Routers (LSR). LERs are located at the edges of the MPLS network. They are responsible for assigning a label to an incoming data packet, and also for removing the label from packets leaving the network. Other nodes in the MPLS network are LSRs. Those are responsible for forwarding packets, using the label from each incoming packet to select an appropriate outgoing link and next LSR (or LER) and the label to be included in the packet sent to that node.

Traffic engineering [19] is the process of controlling how traffic flows through one's network so as to optimize resource utilization and network performance. Traffic engineering is also designed to overcome such problems in the network like link congestion or even link failure by introducing several mechanisms like Fast Reroute (FRR) [20]. FRR is a mechanism for protecting the network from link and node failures by locally repairing the LSPs tunnel at the point of failure, allowing data to continue to flow while the head-end routers attempt to establish a new end-to-end LSPs tunnel to replace the old one. FRR locally repairs the protected LSPs tunnel by rerouting the data over backup tunnels that bypass failed links or nodes.

\section{MPLS-TE BASED PMIPv6 NETWORK ARCHITECTURE}

OMNET++ [14] is a component-based, modular, open-architecture discrete event simulation framework. It provides component architecture for models programmed in $\mathrm{C}++$ [21]. Instead of providing explicit hardwired support for particular computer networks, it provides an infrastructure for writing such simulations. Specific application areas are catered to by various frameworks. One of those is INET-2.0.0 [22] which introduces a simulation of the MIPv6 protocol called xMIPv6.

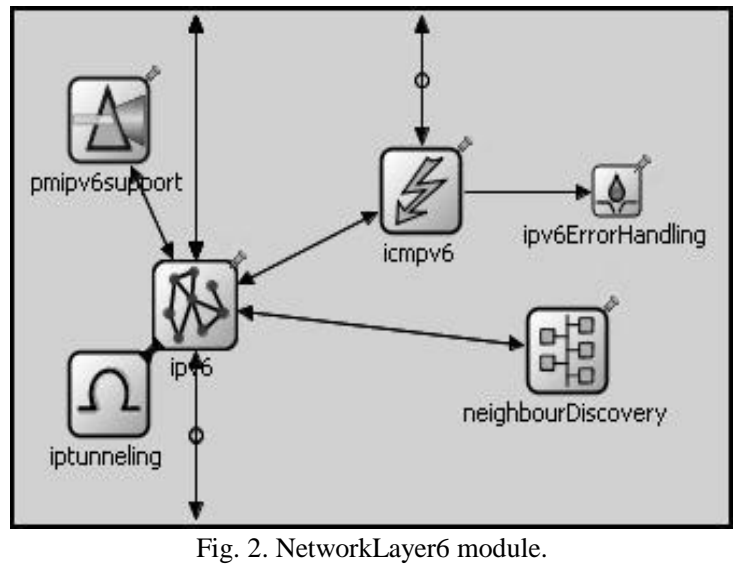

XMIPv6 was implemented by Yousaf et al. [23] and closely follows the IETF's MIPv6 protocol. We have used this as a base, and modified it to support the PMIPv6 protocol.

The Local Mobility Anchor (LMA) module, and Mobile Access Gateway (MAG) module were both created based upon the router6 module from [23]. These support the standard PMIPv6 functions, and become part of the NetworkLayer6 module as illustrated in Fig. 2. All of the PMIPv6 functions will be handled by the PMIPv6 module that is composed of buList, bindingCache, and pmipv6 sub-modules as shown in Fig. 3. 


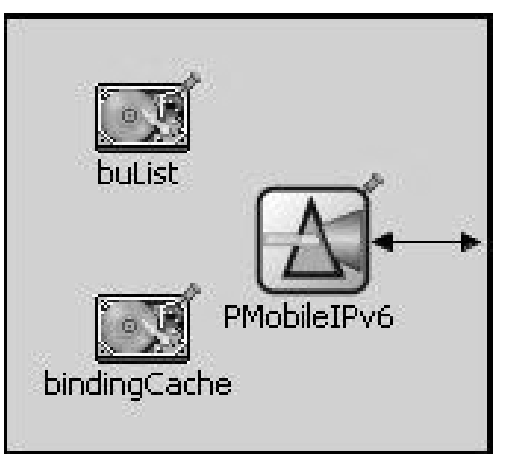

Fig. 3. PMIPv6 module.

MPLS-TE support in OMNET++ also required enhancement to support IPv6, and so support use for PMIPv6. MPLS-TE with integrated IPv6 is added to the LMA and the MAG modules in order to support the MPLS-TE/PMIPv6 functionality, as shown in Fig. 4.

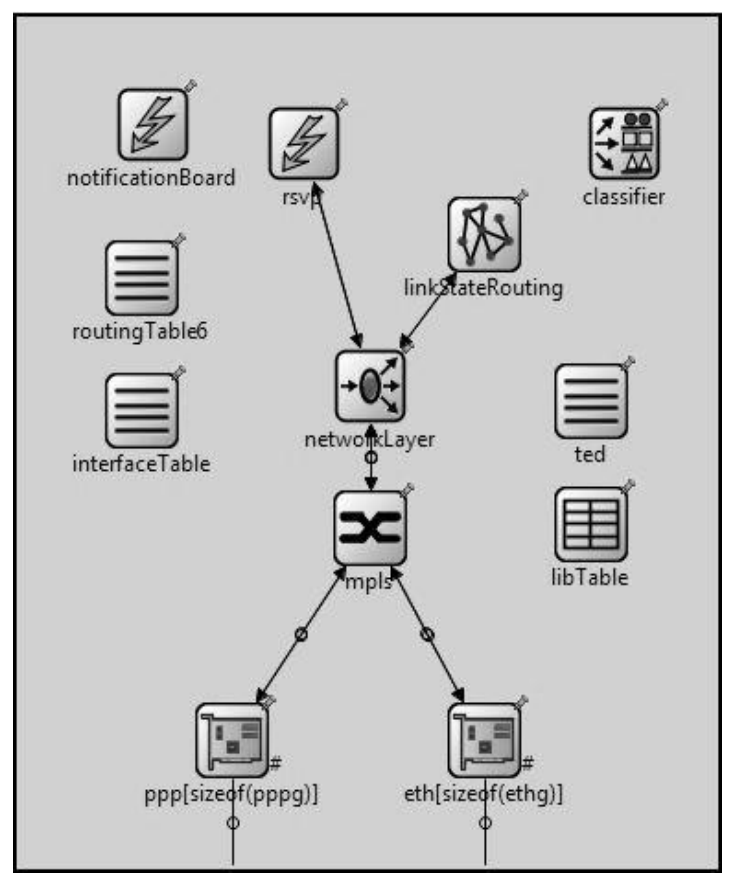

Fig. 4. MPLS-TE based PMIPv6 network module.

\section{PERformanCE EVALUATION AND ANALYSIS}

This research studies more impacts of introducing an LSP tunnel into a PMIPv6 network by studying the end-to-end delay between two edge routers while the mobile node and correspondent node are in the same PMIPv6 network. And we also study the case of link or node failure occurs in the PMIPv6 network in order to analyze the link recovery and continue on forwarding packets.

For every scenario, all network entities are connected with $100 \mathrm{Mbps}$ links with two milliseconds delay each. The mobile node uses a 54Mbps wireless link to connect the access point. The scenarioManager, Configurator, failureManager and channelControl OMNET++ modules are used to support the simulation.

\section{A. End-to-End Delay within the PMIPv6 Network}

End-to-End delay is measured since the 52byte ping message from the mobile node enters the PMIPv6 domain until that ping message exits the PMIPv6 domain to the correspondent node in order to find the time that the message spends in the PMIPv6 domain.

PMIPv6 networks are under common management, so we anticipate that a typical network diameter would be between 3 and 6 hops. For this simulation we consider all of the cases from 2 to 10 hop diameter networks. We also simulate the cases of 12,15 and 20 hops in order to study the trend of the result even though they are rarely happened for the realistic localized network.

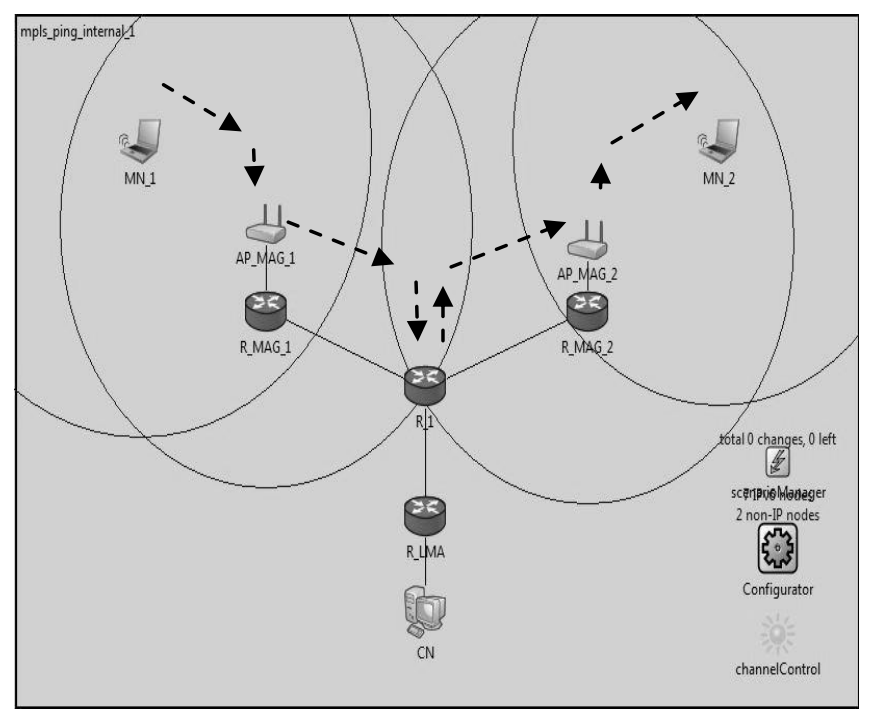

Fig. 5. MN_1 sends ping message to MN_2.

In case of the mobile node and correspondent node are in the same PMIPv6 network, no matter attaching the same MAG or different MAG, the data messages from the mobile node are forwarded to the LMA as shown in Fig. 5.

The end-to-end delay is measured since the R_MAG_1 receives messages from $M N \_1$ until the $\mathrm{R} \_M A G \_2$ sends that messages to MN_2 (R_MAG_1 to R_MAG_2). Or can be measured since the $\mathrm{R} \_M A G \_2$ receives reply messages from MN_2 until the R_MAG_1 sends that messages to MN_1 (R_MAG_2 to R_MAG_1) and the result is shown below.

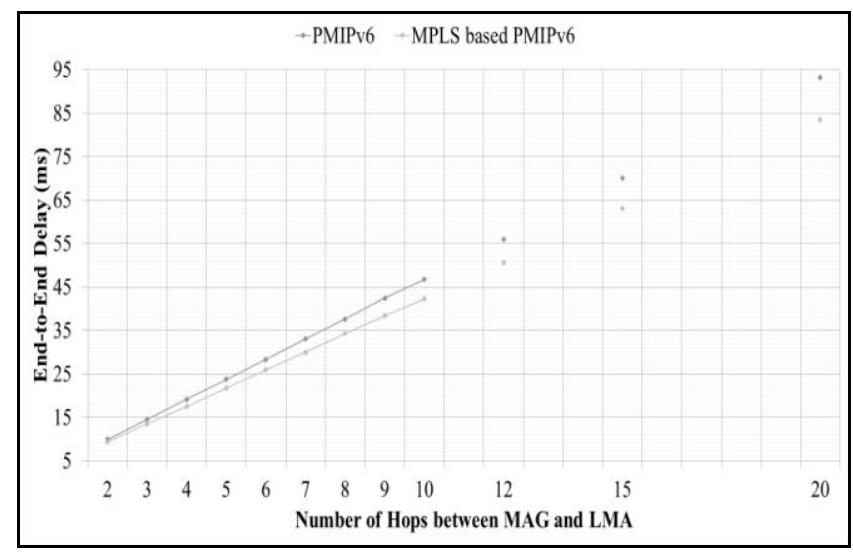

Fig. 6. The result of measuring internal end-to-end delay.

Fig. 6 show the result of measuring end-to-end delay for the case of the mobile node and correspondent node are in the same PMIPv6 network and both attach the same MAG and different MAG. The result shows that introducing LSP tunnel into the PMIPv6 network provides less delay and also provides less increasing rate as the network diameter increases. 


\section{B. Link Recovery}

The case of link or node failure occurs within the PMIPv6 network is simulated in order to study the time to recover the network. There are three types of network to simulate, standard PMIPv6 network, MPLS based PMIPv6 network and MPLS-TE based PMIPv6 network. All networks use the link state routing protocol to discover the failure network with the interval of hello message at 0.1 seconds and hello time out should not exceed 0.15 seconds. And there are four scenarios to simulate which the network diameter to recover are five hops, six hops, seven hops and eight between the MAG and LMA.

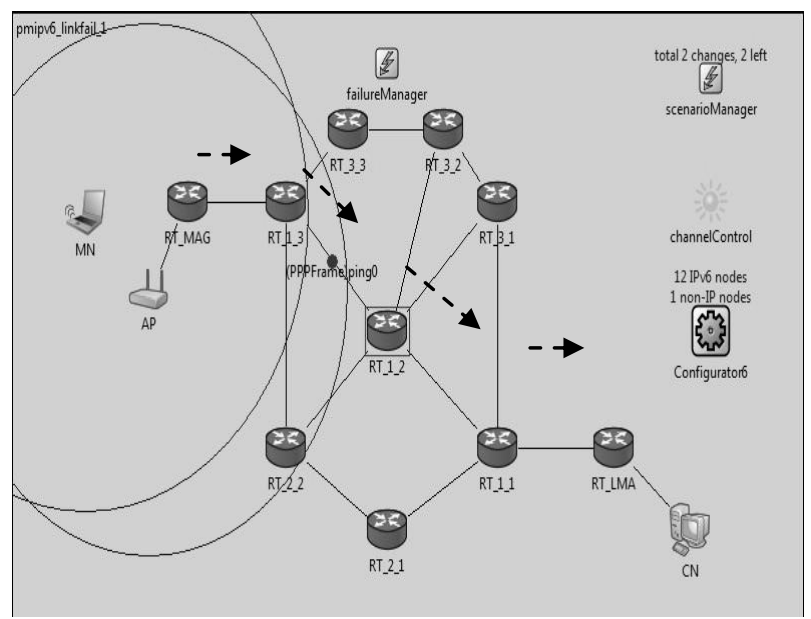

Fig. 7. The shortest path between MAG and LMA.

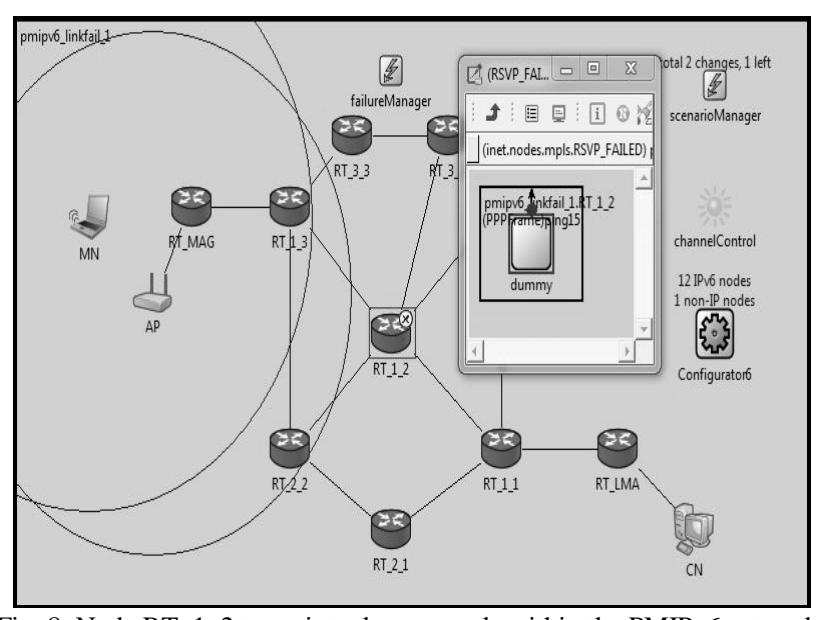

Fig. 8. Node RT_1_2 turns into dummy node within the PMIPv6 network.

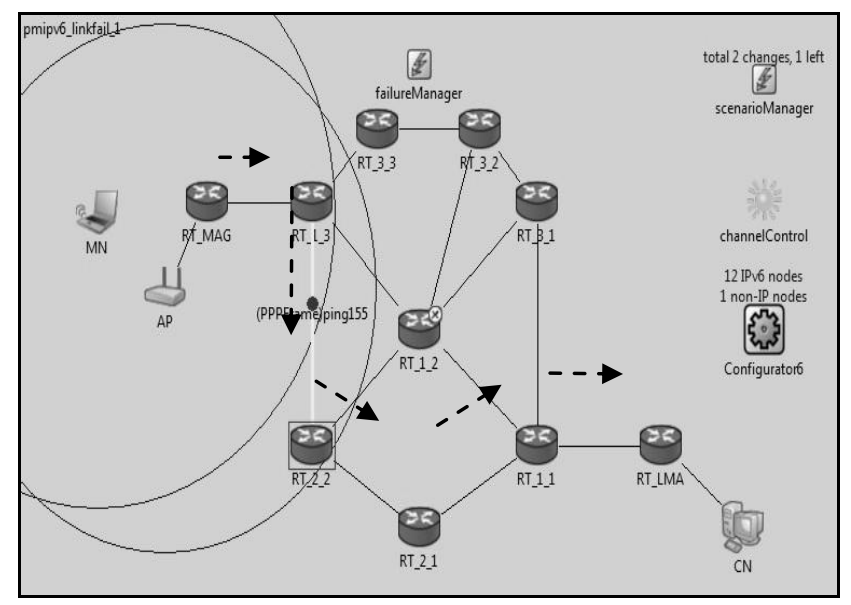

Fig. 9. New recovered shortest path after link recovery.
Fig. 7 illustrates forwarding ping message via the shortest path between MAG and LMA (four hops) and Fig. 8 illustrates the network which the intermediate router RT_1_2 turns into dummy node and unable to route any incoming packet. So, the network waits for link state protocol to detect RT_1_2 failure and updates the new shortest path (five hops) as show in Fig. 9.

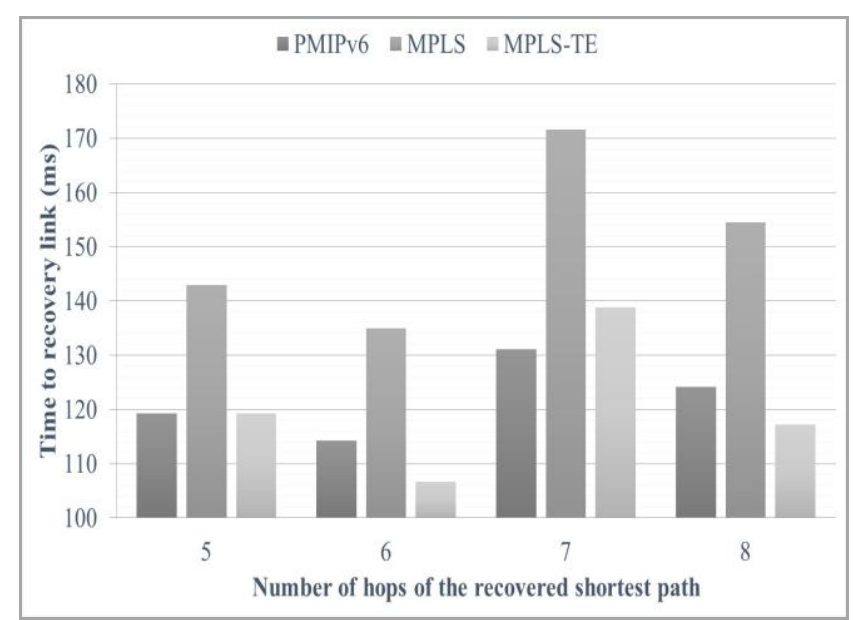

Fig. 10. Result of measuring time to recover link failure.

Fig. 10 show the result of measuring time to recover link in case of link or node failure within the PMIPv6 network which has link state as the protocol to detect link or node failure. The result shows that the standard PMIPv6 and MPLS-TE network does not show much difference because of the same protocol to detect link failure with random simulated hello timeout detect link failure. But for MPLS network, it needs to re-establish an LSP tunnel between the recovered shortest path between MAG and LMA where as the standard PMIPv6 network update new out interface and the MPLS-TE uses the back-up link while re-establishing new LSP tunnel instead.

\section{CONCLUSION AND FUTURE WORK}

This research evaluates more scenarios of using an LSP tunnel instead of IP-in-IP tunnel as would be used by a standard PMIPv6 network. We have also studied the impact of the use of MPLS-TE which provides fast reroute to detect link failure.

The results show that using the LSP tunnel between the MAG and LMA introduces less end-to-end delay for both cases of the mobile node and correspondent node attach the same and different MAG. And introducing fast reroute from the MPLS-TE provides tunnel backup when link failure occur whereas the MPLS tunnel needs more time to establish a new LSP tunnel. So, from what we have studied to date, MPLS-TE/PMIPv6 looks to be a clear winner.

However, localized routing in case of the mobile node and correspondent node are in the same PMIPv6 network can shorten the end-to-end delay which the MAG does not send the packet to LMA, but it knows which MAG that the correspondent node attaches but it is not included in this report. However the results are not yet available, we continue to prove and simulate this in order to shorten the traveling time within the PMIPv6 network. 


\section{REFERENCES}

[1] C. E. Perkins, D. B. Johnson, and J. Arkko, "Mobility support in IPv6," RFC 6275, July 2011.

[2] H. Yokota, K. Chowdhury, R. Koodli, and B. Patil, "Fast handovers for proxy Mobile IPv6,” RFC 5949, September 2010.

[3] H. Soliman, K. Elmalki, and L. Bellier, "Hierrachical mobile IPv6 (HMIPv6) mobility management," RFC 5380, October 2008.

[4] J. Postel, "Internet protocol," RFC 791, September 1981.

[5] C. E. Perkins, "IP mobility support for IPv4," RFC 5944, November 2010.

[6] B. Patil, P. Roberts, C. E. Perkins, and P. R. Calhoun, "Mobile IP network access identifier extension for IPv4," RFC 2794, March 2000

[7] K. Leung, G. Dommety, V. Narayanan, and A. Petrescu, "Network mobility (NEMO) extensions for mobile IPv4," RFC 5177, April 2008.

[8] S. Gundavelli, K. Leung, V. Devarapalli, K. Chowdhury, and B. Patil, "Proxy mobile IPv6," RFC 5213, August 2008.

[9] L. Zhao, J. Zhang, X. J. Zhang, and Q. B. Li, "A mobility management based on proxy MIPv6 and MPLS in aeronautical telecommunications

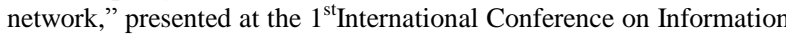
Science and Engineering, December 2009.

[10] R. G. Garroppo, S. Giordano, and L. Tavanti, "Network-based micro-mobility in Wireless Mesh Networks: is MPLS convenient?" GLOBECOM, 2009.

[11] C. A. Astudillo, O. J. Calderon, and J. H. Ortiz, "PM2PLS: an integration of proxy mobile IPv6 and MPLS," International Journal of Computer Science Issues, vol. 8, issue 3, no. 1, May 2011.

[12] R. F. Xia and B. Sarikaya, "MPLS tunnel support for proxy mobile IPv6," IETF Draft, October 25, 2008.

[13] H. Payappanon, T. Kamolphiwong, and K. R. Elz, "Simulation and evaluation of MPLS based PMIPv6 network," presented at International Conference on Computing, Engineering and Communication Technologies, July 2013.
[14] A. Varga. (October 2013). OMNeT++ network simulation framework. [Online]. Available: http://www.omnetpp.org

[15] T. Narten, E. Nordmark, W. A. Simpson, and H. Soliman, "Neighbor discovery for IP version 6 (IPv6)," RFC 4861, September 2007.

[16] S. Thomson, T. Narten, and T. Jinmei, "IPv6 stateless address autoconfiguration," RFC 4862, September 2007.

[17] E. Rosen, A. Viswanathan, and R. Callon, "Multiprotocol label switching architecture," IETF RFC 3031 (Proposed Standard), January 2001.

[18] Deploying IP/MPLS in Mobile Networks, Alcatel-Lucent, 2010.

[19] D. O. Awduche, L. Berger, D. H. Gan, T. Li, V. Srinivasan, and G. Swallow, "RSVP-TE: extensions to RSVP for LSP tunnels," RFC 3209, December 2001.

[20] G. Swallow, P. Pan, and A. Atlas, "Fast reroute extensions to RSVP-TE for LSP tunnels," RFC 4090, May 2005.

[21] J. Soulie. (June 2007). C++ language tutorial. [Online]. Available: http://www.cplusplus.com/doc/tutorial

[22] A. Varga. (October 2013). INET framework | main/welcome to the INET framework! [Online]. Available: http://inet.omnetpp.org

[23] F. Z. Yousaf, C. Bauer, and C. Weitfeld, "An accurate and extensible mobile IPv6 (xMIPv6) simulation model for OMNeT++," in Proc. the $I^{\text {st }}$ International Conference on Simulation Tools and Techniques for Communications, Networks and Systems, Marseille, France, March 2008.

Hatsadin Payappanon received his B.Eng. degree in telecommunication engineering from King Mongkut's Institute of Technology Ladkrabang, Bangkok, Thailand, in 2010. Currently, he is a master student and also a member of Centre for Network Research (CNR) group in Computer Engineering at Prince of Songkla University, Songkla, Thailand. His research interest is computer network and mobility. 